\title{
Customary Broadcast Encryption with Advanced Encryption and Short ciphertexts
}

\author{
Jae Yoon Lee1), Mounika Durbha2)
}

\begin{abstract}
Customary broadcast encryption (BE) plans permit a sender to safely communicate to any subset of individuals however require a trusted gathering to appropriate decoding keys. Group key Authentication (GKA) conventions empower a gathering of individuals to arrange a typical encryption key by means of open systems so that lone the gathering individuals can unscramble the ciphertexts scrambled under the mutual encryption key, yet a sender can't reject a specific part from decoding the ciphertexts. In this paper, we connect these two ideas with a mixture primitive alluded to as contributory communicate encryption (ConBE). In this new primitive, a gathering of individuals arrange a typical open encryption key while every part holds a decoding key. A sender seeing people in general gathering encryption key can confine the decoding to a subset of individuals from his decision. Taking after this model, we propose a ConBE plot with short ciphertexts. The plan is ended up being completely agreement safe under the choice n-Bilinear Diffie-Hellman Exponentiation (BDHE) supposition in the standard model. Of autonomous intrigue, we exhibit another $\mathrm{BE}$ plan that is aggregatable. The aggregatability property is appeared to be helpful to build advanced protocols.
\end{abstract}

Keywords: broadcast encryption, group key agreement, contributory broadcast encryption, provable security, ciphertext.

\section{Introduction}

With the quick progress and unavoidable organization of communication technologies, there is an expanding interest of adaptable cryptographic primitives to secure gathering interchanges and calculation stages. These new stages incorporate texting devices, community oriented registering, portable specially appointed systems and informal organizations. These new applications call for cryptographic primitives permitting a sender to safely scramble to any subset of the clients of the administrations without depending on a completely put stock in

Received(January 29, 2016), Review Result(1st: February 16, 2016, 2nd: March 17, 2016), Accepted(June 10, 2016)

${ }^{1}$ Department of Applied Computer Engineering, Dankook University, 152 Jukjeon-ro, Suji-gu, Gyeonggi-do email: ultrastory@gmail.com

${ }^{2}$ (Corresponding Author) Department of Computer Science and Engineering, KL University email: mounika.dr96@gmail.com 
merchant. Communicate encryption (BE) [1] is a very much concentrated primitive proposed for secure gathering focused correspondences. It permits a sender to safely communicate to any subset of the gathering individuals. By and by, a BE framework vigorously depends on a completely trusted key server who creates mystery decoding keys for the individuals and can read every one of the correspondences to any individuals.

Group key agreement (GKA) is another surely knew cryptographic primitive to secure gathering focused correspondences. A traditional GKA [2] permits a gathering of individuals to set up a typical mystery key by means of open systems. In any case, at whatever point a sender needs to make an impression on a gathering, he should first join the gathering and run a GKA convention to impart a mystery key to the expected individuals. All the more as of late, and to beat this constraint, $\mathrm{Wu}$ et al. presented unbalanced GKA [3], in which just a typical gathering open key is arranged and every gathering part holds an alternate decoding key. Nonetheless, neither traditional symmetric GKA nor the recently presented awry GKA permit the sender to singularly avoid a specific part from perusing the plaintext. Hence, it is essential to find more flexible cryptographic primitives allowing dynamic broadcasts without a fully trusted dealer[4-6].

\section{Proposed System}

\subsection{Related Work}

Various works have tended to key understanding conventions for different gatherings. The plans because of Ingemarsson et al. [2] and Steiner et al. [7] are intended for $\mathrm{n}$ parties and require $\mathrm{O}(\mathrm{n})$ rounds. Tree key structures have been further proposed, diminishing the quantity of rounds to $\mathrm{O}(\operatorname{logn})$ [8-10]. Multi-round GKA conventions represent a synchronism prerequisite: keeping in mind the end goal to finish the convention, all the gathering individuals need to remain online at the same time. Step by step instructions to advance the round unpredictability of GKA protocols has been contemplated in a few works (e.g., [11-13]). In [14], Tzeng introduced a consistent round GKA convention that can distinguish con artists. Thusly, Yi [15] developed a blame tolerant convention in a personality based setting. Burmester and Desmedt [16] proposed a two-round n-party GKA convention for $n$ parties. The Joux convention [17] is one-round and just material to three gatherings. The work of Boneh and Silverberg [18] demonstrates a one-round $(n+1)$ - party GKA convention with n-linear pairings.

Dynamic GKA protocols give additional instruments to deal with part changes. Bresson et al. 
[19-20] extended the convention in [21] to dynamic GKA protocols that permit individuals to leave and join the gathering. The quantity of rounds in the set-up=join calculations of the Bresson et al's. conventions [19-20] is direct with the gathering size, however the quantity of rounds in the leave calculation is consistent. The hypothetical examination in [22] demonstrates that for any tree-based gathering key understanding plan, the lower bound of the most pessimistic scenario cost is $\mathrm{O}(\log )$ rounds of collaboration for a part to join or leave. Without depending on a tree-based structure, Kim et al. [23] proposed a two-round element GKA protocol. As of late, Abdalla et al. [24] exhibited a two-round element GKA convention in which just a single round is required to adapt to the change of individuals on the off chance that they are in the underlying gathering. Jarecki et al. [25] exhibited a powerful two-round GKA convention in which a session key can be built up regardless of the possibility that a few members fall flat amid the execution of the convention. Watching that current GKA conventions can't deal with sender/part changes effectively, $\mathrm{Wu}$ et al. displayed a gathering key administration protocol [26-28] in which a change of the sender or monotone avoidance of gathering individuals does not require additional correspondence, and changes of different individuals require one additional round.

\subsection{Existing System}

Group key agreement (GKA) is another well-understood cryptographic primitive to secure gathering focused correspondences. A routine GKA permits a gathering of individuals to build up a typical mystery key by means of open systems. In any case, at whatever point a sender needs to make an impression on a gathering, he should first join the gathering and run a GKA convention to impart a mystery key to the expected individuals.

More as of late, and to beat this impediment, $\mathrm{Wu}$ et al. presented deviated GKA, in which just a typical gathering open key is arranged and every gathering part holds an alternate decoding key.

However, neither conventional symmetric GKA nor the recently presented deviated GKA permit the sender to singularly bar a specific part from perusing the plaintext. Consequently, it is basic to discover more adaptable cryptographic primitives permitting dynamic communicates without a completely trusted dealer.

\subsubsection{Disadvantage of Existing System}


- Need a fully trusted third party to set up the system.

- Existing GKA protocols cannot handle sender/member changes efficiently.

\subsection{Proposed System}

We introduce the Contributory Broadcast Encryption (ConBE) primitive, which is a half and half of GKA and BE.

This full paper gives finish security proofs, represents the need of the aggregatability of the basic BE building square and demonstrates the reasonableness of our ConBE plot with experiments.

First, we show the ConBE primitive and formalize its security definitions. ConBE consolidates the basic thoughts of GKA and BE. A gathering of individuals connect by means of open systems to arrange an open encryption key while every part holds an alternate mystery decoding key. Utilizing people in general encryption key, anybody can scramble any message to any subset of the gathering individuals and just the planned collectors can decode.

We formalize plot resistance by characterizing an aggressor who can completely control every one of the individuals outside the expected collectors however can't remove helpful data from the ciphertext.

Second, we exhibit the idea of aggregatable communicate encryption (AggBE). Coarsely, a BE plan is aggregatable if its safe examples can be amassed into another protected occasion of the BE plan. In particular, just the collected unscrambling keys of a similar client are substantial decoding keys comparing to the accumulated open keys of the hidden BE occurrences.

Finally, we develop an effective ConBE plot with our AggBE conspire as a building square. The ConBE development is turned out to be semi-adaptively secure under the choice BDHE presumption in the standard model.

\subsubsection{Advantages of Proposed System}

We construct a concrete AggBE scheme tightly proven to be fully collusion-resistant under the decision BDHE assumption.

The proposed AggBE scheme offers efficient encryption/decryption and short ciphertexts.

Only one round is required to establish the public group encryption key and set up the ConBE system. 


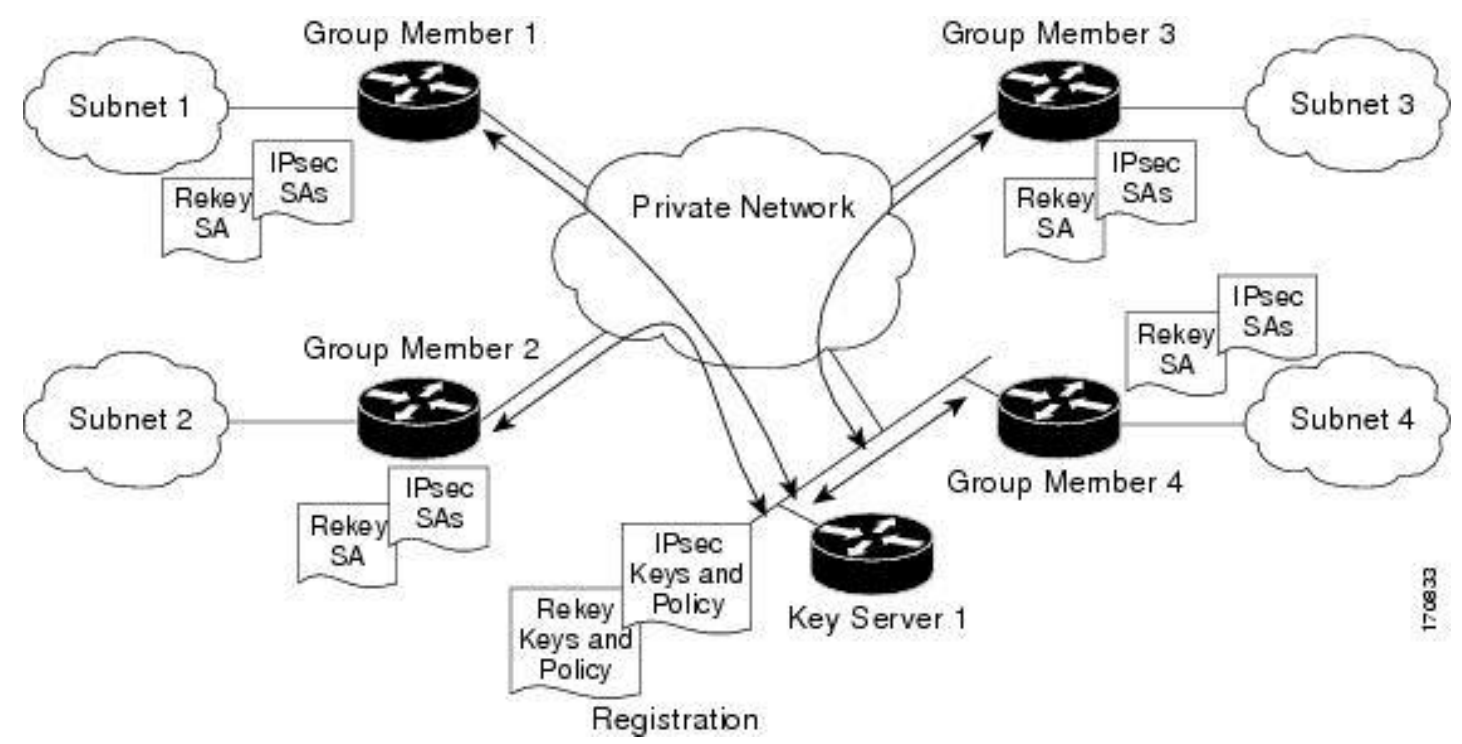

[Fig 1] System Architecture

\section{CONCLUSION}

In this paper, we formalized the ConBE primitive. In ConBE, anybody can send mystery messages to any subset of the gathering individuals, and the framework does not require a trusted key server. Neither the change of the sender nor the dynamic decision of the proposed beneficiaries require additional rounds to arrange aggregate encryption/unscrambling keys. Taking after the ConBE display, we instantiated a productive ConBE conspire that is secure in the standard model. As a flexible cryptographic primitive, our novel ConBE thought opens another road to build up secure communicate channels and can be required to secure various rising distributed computation applications.

\section{References}

[1] A. Fiat and M. Naor, Broadcast encryption, Proc. Crypto, (1993), pp.480-491.

[2] I. Ingemarsson, D. T. Tang and C. K. Wong, A conference key distribution system, IEEE Trans. Inf. Theory, (1982), Vol.28, No.5, pp.714-720.

[3] Q. Wu, Y. Mu, W. Susilo, B. Qin and J. Domingo-Ferrer, Asymmetric group key agreement, Proc. Eurocrypt, (2009), pp.153-170. 
[4] http://en.wikipedia.org/wiki/PRISM_\%28surveillance_program\%29, May 5 (2014).

[5] Q. Wu, B. Qin, L. Zhang, J. Domingo-Ferrer and O. Farr, Bridging broadcast encryption and group key agreement, Proc. 17th Int. Conf. The Theory Appl. Cryptol. Inform. Secur., (2011), pp.143-160.

[6] D. H. Phan, D. Pointcheval and M. Strefler, Decentralized dynamic broadcast encryption, Proc. 8th Int. Conf. Secur. Cryptography Netw., (2011), pp.166-183.

[7] M. Steiner, G. Tsudik and M. Waidner, Key agreement in dynamic peer groups, IEEE Trans. Parallel Distrib. Syst., (2000), Vol.11, No.8, pp.769-780.

[8] A. Sherman and D. McGrew, Key establishment in large dynamic groups using one-way function trees, IEEE Trans. Softw. Eng., (2003), Vol.29, No.5, pp.444-458.

[9] Y. Kim, A. Perrig and G. Tsudik, Tree-based group keyagreement, ACM Trans. Inf. Syst. Secur., (2004), Vol.7, No.1, pp.60-96

[10] Y. Mao, Y. Sun, M. Wu and K. J. R. Liu, JET: Dynamic Join-Exit-Tree Amortization and Scheduling for Contributory Key Management, IEEE/ACMTrans. Netw., (2006), Vol.14, No.5, pp.1128-1140.

[11] C. Boyd and J. M. Gonz_alez-Nieto, Round-optimal contributory conference key agreement, Proc. 6th Int. Workshop Pract. Theory Public Key Cryptography, (2003), pp.161-174.

[12] W. G. Tzeng and Z. J. Tzeng, Round efficient conference key agreement protocols with provable security, Proc. 6th Int. Conf.Theory Appl. Cryptol. Inform. Secur.: Adv. Cryptol., (2000), pp.614-627.

[13] R. Dutta and R. Barua, Provably secure constant round contributory group key agreement in dynamic setting, IEEE Trans. Inf. Theory, (2008), Vol.54, No.5, pp.2007-2025.

[14] W. G. Tzeng, A secure fault-tolerant conference-key agreement protocol, IEEE Trans. Comput., (2002), Vol.51, No.4, pp.373-379.

[15] X. Yi, Identity-based fault-tolerant conference key agreement, IEEE Trans. Dependable Secure Comput. (2004), Vol.1, No.3, pp.170-178.

[16] M. Burmester and Y. Desmedt, A secure and efficient conference key distribution system in Proc. Workshop Theory Appl. Cryptographic Techn., (1994), pp.275-286.

[17] A. Joux, A one round protocol for tripartite Diffie-Hellman, J. Cryptol., (2004), Vol.17, No.4, pp.263-276.

[18] D. Boneh and A. Silverberg, Applications of multilinear forms tocrytography Contemp. Math., (2003), Vol. 324, pp.71-90.

[19] E. Bresson, O. Chevassut and D. Pointcheval, Provably authenticatedgroup Diffie-Hellman key exchangeThe dynamic case, Proc. Conf. Theory Appl. Cryptol. Inform. Secur.: Adv. Cryptol., (2001), pp.290-309.

[20] E. Bresson, O. Chevassut and D. Pointcheval, Dynamic group Diffie-Hellman key exchange under standard assumptions, Proc. Int. Conf. Theory Appl. Cryptographic Techn., (2002), pp.321-336.

[21] E. Bresson, O. Chevassut, D. Pointcheval and J. J. Quisquater, Provably authenticated group Diffie-Hellman key exchange, Proc. ACM Comput. Commun. Secur., (2001), pp.255-264.

[22] J. Snoeyink, S. Suri and G. Varghese, A lower bound for multicastkey distribution, Proc. 20th Annu. Joint 
Conf. IEEE Comput.Commun. Soc., (2001), pp.422-431.

[23] H. J. Kim, S. M. Lee and D. H. Lee, Constant-round authenticated group key exchange for dynamic groups, Proc. Conf. Theory Appl. Cryptol. Inform. Secur.: Adv. Cryptol., (2004), pp.245-259.

[24] M. Abdalla, C. Chevalier, M. Manulis and D. Pointcheval, Flexible group key exchange with on-demand computation of subgroupkeys, Proc. 3rd Int. Conf. Cryptol. Africa, (2010), pp.351-368.

[25] S. Jarecki, J. Kim and G. Tsudik, Flexible robust group keyagreement IEEE Trans. Parallel Distrib. Syst., (2011), Vol.22, No.5, pp.879-886.

[26] Q. Wu, B. Qin, L. Zhang, J. Domingo-Ferrer and J. Manj_on, Fast transmission to remote cooperative groups: A new key management paradigm, IEEE/ACM Trans. Netw., (2013), Vol.21, No.2, pp.621-633.

[27] E. Bertino, N. Shang, and S. S. Wagstaff Jr., An efficient time bound hierarchical key management scheme for secure broadcasting, IEEE Trans. Dependable Secure Comput., (2008), Vol.5, No.2, pp.65-70.

[28] A. Shoufan and S. A. Huss, High-performance rekeying processor architecture for group key management IEEE Trans. Comput., (2009), Vol.58, No.10, pp.1421-1434. 
Customary Broadkast Encryption with Advanced Encryption and Short ciphertexts

(This page is empty intentionally) 